\title{
Microvolt T-wave alternans in adults with complex congenital heart diseases
}

\author{
Olga Trojnarska ${ }^{1}$, Aleksandra Ciepłucha ${ }^{1}$, Agnieszka Bartczak ${ }^{1}$, \\ Lucyna Kramer², Stefan Grajek ${ }^{1}$ \\ ${ }^{1} 1^{\text {st }}$ Department of Cardiology, University of Medical Sciences, Poznan, Poland \\ ${ }^{2}$ Department of Computer Sciences and Statistics, University of Medical Sciences, Poznan, Poland
}

\begin{abstract}
Background: Sudden cardiac death (SCD) mostly resulting from ventricular arrhythmia remains a cause of mortality in 19-30\% of adults with congenital heart defects. Indications for implantable cardioverter-defibrillators in primary prophylaxis are still under research. Microvolt T wave alternans (MTWA) is one of the SCD risk stratification methods. We determined the incidence of MTWA in these patients and its coincidence with ventricular arrhythmia, as well as risk factors of ventricular arrhythmia/SCD.
\end{abstract}

Methods: 204 patients with complex congenital heart anomalies and 45 healthy volunteers underwent ambulatory ECG monitoring, a cardiopulmonary test, B-type natriuretic peptide assessment, echocardiography and an MTWA test. After excluding technically inadequate traces, the remaining 179 patients and 43 controls were classified into MTWA positive (+), negative (-) and indeterminate (ind) subgroups. Additionally, MTWA (+) and MTWA (ind) formed an 'abnormal' group, labeled MTWA (non-).

Results: Abnormal MTWA was observed more frequently in the study group compared to controls (59 [33.0\%] vs. 1 [2.3\%], $p=0.000001$ ). The MTWA (non-) group compared to MTWA (-) presented a higher number of males (61.0\% vs. $37.5 \%, p=0.005)$, predominance of patients with NYHA > I (44.1\% vs. $25.0 \%, p=0.007)$, pulmonary hypertension $(16.9 \%$ vs. $0.8 \%, p=0.00007$ ), lower blood saturation (97\% [73-100] vs. 99\% [69-100], $p=0.0003$ ), higher incidence of malignant arrhythmia (9 [15.2\%] vs. 3 [2.5\%], $p=0.003)$, lower peak oxygen consumption $\mathrm{VO}_{2}[\mathrm{~mL} / \mathrm{kg} / \mathrm{min}](23.1 \pm 5.9$ vs. $26.3 \pm 6.7, p=0.002)$, higher $\mathrm{VE} /$ $/ \mathrm{VCO}_{2}$ slope (36.0 [25-74] vs. 31.0 [21-58], $p=0.01$ ). Multivariate logistic regression analysis proved that pulmonary hypertension $(O R=13.7, p=0.03)$, male gender $(O R=10.4$, $p=0.00002), \mathrm{VE} / \mathrm{VCO}_{2}$ slope $(\mathrm{OR}=1.07, p=0.045)$ and $\mathrm{VO}_{2}(\mathrm{OR}=0.89, p=0.04) \mathrm{in}$ crease the probability of MTWA (non-).

Conclusions: Abnormal MTWA is more frequent in adults with congenital heart diseases than in the healthy population. Its probability increases in patients demonstrating clinical findings conducive to lethal arrhythmia like heart failure and pulmonary hypertension. (Cardiol J 2014; 21, 2: 144-151)

Key words: congenital heart disease, T-wave alternans, sudden cardiac death, ventricular arrhythmia, systemic right ventricle, univentricular heart, tetralogy of Fallot

Address for correspondence: Aleksandra Ciepłucha, MD, PhD, $1^{\text {st }}$ Department of Cardiology, University of Medical Sciences, ul. Długa 1/2, 61-848 Poznań, Poland, tel: +48 6185491 46, fax: +48 61854 90 94, e-mail: ola.cieplucha@gmail.com 


\section{Introduction}

Sudden cardiac death (SCD), mostly resulting from ventricular arrhythmia, remains a cause of mortality in $19-30 \%$ of adults with congenital heart defects [1-5]. Such dramatic events in this relatively young population prompt to investigate indications for implantable cardioverter-defibrillator (ICD) therapy in primary prophylaxis. The mechanisms leading to ventricular arrhythmia in these patients are still under research. However, ventricular arrhythmia is considered to result from myocardial fibrosis caused by cyanosis and/or inadequate intraoperative cardioprotection, as well as scars after ventriculotomy. Moreover, hemodynamic changes leading to dilation of ventricles (or single ventricle) and a consequently increased wall stress resulting in areas of electrical inhomogeneity within myocardium also contribute to these rhythm disturbances [3-8]. All mentioned pathological phenomena may additionally lead to heart failure which is a known cause of malignant arrhythmia and SCD per se $[5,6,8,9]$. The decision on ICD therapy is often based on electrophysiology study which has demonstrated better prognostic value when conducted in preselected group of patients $[6,10]$. Such preselection of subjects potentially endangered with SCD is performed with the use of some noninvasive methods, including a microvolt T wave alternans (MTWA) — an electrocardiographic (ECG) phenomenon displaying inhomogeneity of the myocardial repolarization process. It was proved that the inhomogeneity of the intracardiac repolarization leads to a dispersion of the depolarizing wave, re-entry phenomenon and, as a consequence, initiation of the ventricular arrhythmia [9, 11, 12]. A prognostic value of MTWA was strongly suggested among patients with ischemic and nonischemic advanced heart failure [13-16]. To our knowledge, the issue of spectral MTWA has been addressed in merely
2 papers regarding pediatric population and only 1 concerning adults with congenital heart diseases (CHD) characterized by right ventricle pathology or single ventricle physiology that was conducted by our team [17-19]. Therefore, a necessity to continue this research among grown-up patients with other congenital cardiac anomalies seems undeniable.

The aim of the study was to evaluate the incidence of spectral MTWA in adults with CHD; to determine its coincidence with malignant and potentially malignant arrhythmia, as well as other clinical and demographical findings conducive to malignant arrhythmia and SCD in the study group.

\section{Methods}

The study protocol, as approved by the Ethics Committee of the University of Medical Sciences in Poznan, conformed to the ethical guidelines of the 1975 Declaration of Helsinki. Informed consent was obtained from all subjects.

The study group was selected from the population followed-up in 2010-2012 at the Congenital Heart Disease Outpatient Clinic of the $1^{\text {st }}$ Department of Cardiology, University of Medical Sciences in Poznan, Poland. Out of 204 consecutive adults with complex CHD and 45 healthy volunteers, after exclusion of MTWA tests impossible to interpret due to technical artifacts, 179 patients and 43 controls were further analyzed. The patients (81 males), aged 18-75 (median 30 ) years, with $126(70.4 \%)$ being operated at the age of 0.5-21 (median 5) years (Table 1) demonstrated the following defects: $5(5.8 \%)$ - common arterial trunk, $9(5.0 \%)$ - corrected transposition of great arteries, $4(2.2 \%)$ - double outlet right ventricle, $27(15.1 \%)$ - Ebstein anomaly, $8(4.5 \%)$ - Eisenmenger syndrome, $18(10.0 \%)$ - transposition of the great arteries, $105(50.3 \%)$ tetralogy of

Table 1. Demographic and clinical characteristic of the study and control groups.

\begin{tabular}{lccc}
\hline & $\begin{array}{c}\text { Study group } \\
\text { (n = 179) }\end{array}$ & $\begin{array}{c}\text { Control group } \\
\text { (n = 43) }\end{array}$ & $\begin{array}{c}\text { P (study vs. } \\
\text { control group) }\end{array}$ \\
\hline Males & $81(45.3 \%)$ & $22(51.1 \%)$ & 0.5 \\
Age [years]; median (min-max) & $30(18-75)$ & $28(18-65)$ & 0.52 \\
Body mass index [kg/m²]; median (min-max) & $21.8(15.6-38.0)$ & $23.0(17.3-32.0)$ & 0.323 \\
New York Heart Association > I & $56(31.3 \%)$ & $0(0.0 \%)$ & $<0.00001$ \\
Blood oxygen saturation [\%]; median (min-max) & $98(69-100)$ & $100(99-100)$ & $\mathbf{0 . 0 0 0 1}$ \\
Operated & $126(70.4 \%)$ & - & - \\
Age at operation [years]; median (min-max) & $5.0(0.5-21)$ & - & - \\
Pulmonary hypertension & $11(6.1 \%)$ & - & - \\
\hline
\end{tabular}


Fallot, $18(10.0 \%)$ - univentricular heart including: 8 - tricuspid atresia, 3 - corrected transposition, 4 - double inlet left ventricle, 3 - common atrioventricular canal. The control group consisted of 43 healthy volunteers ( 22 males) aged $18-65$ (median 28) years. The main inclusion criterion was sinus rhythm. Exclusion criteria were: persistent atrial fibrillation, permanent ventricular pacing, serum creatinine level $>140 \mu \mathrm{mol} / \mathrm{L}$, serum aspartate transaminase level exceeding twice the upper limit of normal, pulmonary diseases.

Functional status was evaluated according to New York Heart Association (NYHA) classification. Blood oxygen saturation was assessed using pulse oximetry.

Microvolt T wave alternans was analyzed in 204 patients during treadmill test while receiving their usual medications, including $\beta$-blocker therapy [12]. High-resolution electrodes (Micro-V Alternans Sensors, Cambridge Heart Inc.) were applied using Frank orthogonal configuration. MTWA was recorded during a heart rate (HR)-based exercise protocol using CH2000 (Cambridge Heart Inc., Bedford, MA, USA) using spectral method [11]. The test was considered positive if the significant MTWA (i.e. alternans voltage $\geq 1.8 \mu \mathrm{V}$ and alternans ratio $>3.0$ ) appeared at an onset $\mathrm{HR} \leq 110 \mathrm{bpm}$, was present for $\geq 1 \mathrm{~min}$ in any orthogonal lead or 2 adjacent precordial leads and sustained as long as HR remained above the patient specific onset HR. The test was classified as negative if sustained MTWA was absent at HR $\leq 110 \mathrm{bpm}$ and if there was $\geq 1 \mathrm{~min}$ at $\mathrm{HR} \geq 105 \mathrm{bpm}$ of artifact-free trace (noise level $<1.8 \mu \mathrm{V}$, ectopy $<10 \%)$. Otherwise, the test was considered indeterminate and immediately repeated in order to reduce the number of such results. As generally accepted [12], the indeterminate test may be caused either by patient factors (including: 1) excessive ectopy during exercise, 2) unsustained MTWA, 3) failure to achieve $\mathrm{HR}=105 \mathrm{bpm}$ ) or technical factors (including: 1) a noisy recording, 2) failure to maintain HR between 105 and $110 \mathrm{bpm}$ for $\geq 1 \mathrm{~min}$ ). The indeterminate results due to technical factors obtained in 25 patients were ultimately excluded from further analysis, as a consequence the analysis was performed in 179 subjects. Considering the similar prognostic significance, all tests classified as positive or indeterminate due to patient factors were combined into a common group labeled 'nonnegative' [MTWA (non-)], i.e. abnormal. The analysis of MTWA results and statistical calculations were performed by the researchers blinded to all ECG, clinical and laboratory findings.
Echocardiography was performed with VIVID 7 GE Medical System device, using 1.5-2.5 Hz probe in 2-dimensional, $\mathrm{M}$ - and Doppler modes. The diagnoses of anatomical anomalies were set according to the current recommendations of the European Society of Cardiology (ESC) [7]. As in other studies [20], the diagnosis of pulmonary arterial hypertension was based on echocardiography. The estimation of pulmonary arterial pressure (PAP) was based on the peak velocity of the jet of tricuspid regurgitation enabling to calculate right ventricular systolic pressure (using Bernoulli equation). After excluding pulmonary stenosis, PAP was calculated as the sum of right ventricular systolic pressure and right atrial pressure (estimated as $5-15 \mathrm{~mm} \mathrm{Hg}$ depending on the diameter and respiratory variation of the inferior vena cava). According to the current guidelines we considered the criterion of estimated PAP $\geq 37 \mathrm{~mm} \mathrm{Hg} \mathrm{com-}$ bined with additional echocardiographic variables suggestive of pulmonary arterial hypertension a sufficient basis to set this diagnosis [20].

The cardiopulmonary exercise treadmill test was performed according to the modified Bruce protocol (adding stage $0-3 \mathrm{~min}, 1.7 \mathrm{~km} / \mathrm{h}, 5 \%$ grading) and was symptom limited. The equipment was calibrated with a standard gas mixture before each test. Patients were encouraged to continue the exercise until the respiratory exchange ratio (RER) exceeded 1. Oxygen uptake (peak $\mathrm{VO}_{2}$ ), carbon dioxide production $\left(\mathrm{VCO}_{2}\right)$ and minute ventilation were measured with breath by breath technique using Sensor Medics, model $\mathrm{V}_{\max } 29$. PeakVO ${ }_{2}$ was defined as the average for the last $20 \mathrm{~s}$ of exercise and expressed as $[\mathrm{mL} / \mathrm{kg} / \mathrm{min}]$ and $[\mathrm{mL} / \mathrm{min}]$, as well as the percentage ranges predicted for sex, age, height and weight [21]. A ventilation/ /carbon dioxide slope ( $\mathrm{VE} / \mathrm{VCO}_{2}$ slope) was assessed by linear regression for the whole exercise. A standard 12-lead ECG was continuously recorded. Due to physical disability a cardiopulmonary test was not performed in 3 patients.

Ambulatory 24-hour ECG monitoring was registered on the same day as the other tests using CardioScan12.0 Premier, Oxford system. Additionally, all previous results of ambulatory ECG available in the patients' medical records were analyzed for presence of ventricular arrhythmia, which was classified as: excessive ventricular ectopy (ventricular premature beats - VPB $\geq 10 / \mathrm{h}$ ), episodes of non-sustained ventricular tachycardia - nsVT (presence of $\geq 3$ consecutive ventricular beats at a rate greater than $100 \mathrm{bpm}$, terminating spontaneously in less than $30 \mathrm{~s}$ ), episodes of sustained 
Table 2. Microvolt T-wave alternans (MTWA) in the study and control groups.

\begin{tabular}{lccc}
\hline & Study group $(\mathbf{n}=\mathbf{1 7 9})$ & Control group $(\mathbf{n}=\mathbf{4 3})$ & $\mathbf{P}$ (study vs. control group) \\
\hline MTWA (+) & $31(17.3 \%)$ & $1(2.3 \%)$ & $\mathbf{0 . 0 0 8}$ \\
MTWA (ind) & $28(15.6 \%)$ & $0(0.0 \%)$ & $\mathbf{0 . 0 0 3}$ \\
MTWA (non-) & $59(33.0 \%)$ & $1(2.3 \%)$ & $\mathbf{0 . 0 0 0 0 0 1}$ \\
MTWA (-) & $120(67.0 \%)$ & $42(97.7 \%)$ & $\mathbf{0 . 0 0 0 0 1}$ \\
\hline
\end{tabular}

MTWA (+) - positive result; MTWA (ind) — result indeterminate due to patient factors; MTWA (-) — negative result; MTWA (non-) - non-negative (abnormal) result

ventricular tachycardia - VT (ventricular tachycardia greater than $30 \mathrm{~s}$ in duration or hemodynamically unstable) [6]. According to the classification regarding patients with structural heart disease [6] this findings were divided into two groups: potentially malignant ventricular arrhythmia (VPB $>10 / \mathrm{h}$ and/or nsVT) and malignant ventricular arrhythmia (VT). For the purposes of analysis, a group comprising of patients with any of the specified forms of ventricular arrhythmia was formed and labeled 'VT/nsVT/VPB > 10/h'.

QRS duration was adopted as the longest value measured among all 12 leads in standard ECG.

Serum B-type natriuretic peptide (BNP) level was measured in the venous blood samples taken after 15 min of rest in supine position using the Abbott AxSYM Immunoassay system.

\section{Statistical analysis}

Continuous variables were summarized by mean \pm SD or median and minimal-maximal values, depending on normality of distribution assessed with Shapiro-Wilk test. Variables following normal distribution were analyzed using Student's t test or Welch test, otherwise non-parametrical U Mann-Whitney test comparing the two groups was performed. Categorical variables are represented by frequencies and percentages and calculated as $\chi^{2}$ test with Yates's correction or Fisher's exact test. Factors associated with non-negative MTWA were assessed by linear logistic regression. $\mathrm{P}<0.05$ were considered statistically significant.

\section{Results}

The incidence of MTWA (+), MTWA (ind), as well as MTWA (non-) in the study group was significantly higher than among the controls (Table 2).

Comparison between the subgroups with different MTWA results demonstrated male predominance among MTWA (+) and MTWA (non-) patients in contrast to MTWA (-) group. Subjects with MTWA (non-) manifested heart failure symptoms - NYHA > I significantly more often when compared to MTWA (-) subgroup. Blood saturation was higher in MTWA (-) patients than in subgroups with MTWA (+) and MTWA (non-). Pulmonary hypertension was also less common in MTWA (-) group when compared to MTWA (+) and MTWA (non-) (Table 3).

As shown in Table 4 the incidence of malignant (VT) and potentially malignant (nsVT/VPB $>10 / \mathrm{h}$ ) arrhythmia as well as any specified form of ventricular arrhythmia (VT/nsVT/VPB > 10/h) was significantly higher in subjects with MTWA (non-) as compared to MTWA (-).

The cardiopulmonary test revealed significantly lower values of peak oxygen consumption $\left(\mathrm{VO}_{2}\right.$, $[\mathrm{mL} / \mathrm{kg} / \mathrm{min}]$ ) in MTWA (+) and MTWA (non-) subgroups than in MTWA (-) patients. Similar differences were observed in percentage values of maximal predicted oxygen consumption $\left(\% \mathrm{VO}_{2}\right.$, $[\mathrm{mL} / \mathrm{kg} / \mathrm{min}])$, as well as $\% \mathrm{VO}_{2}[\mathrm{~L} / \mathrm{min}]$. Patients demonstrated a higher ventilation/carbon dioxide index $-\mathrm{VE} / \mathrm{VCO}_{2}$ slope and higher serum BNP concentration compared to those with MTWA (-) (Table 5).

As shown in univariate linear logistic regression analysis, the factors increasing probability of abnormal MTWA were: pulmonary hypertension, sustained ventricular arrhythmia, as well as any specified form of ventricular arrhythmia, male gender, NYHA > I, increased $\mathrm{VE} / \mathrm{VCO}_{2}$ slope, abnormal $\mathrm{SO}_{2}$ as well as decreased values of $\% \mathrm{VO}_{2}$ $[\mathrm{mL} / \mathrm{kg} / \mathrm{min}], \% \mathrm{VO}_{2}[\mathrm{~L} / \mathrm{min}]$ and $\mathrm{VO}_{2}[\mathrm{~mL} / \mathrm{kg} / \mathrm{min}]$. A multivariate logistic regression analysis proved a significant predictive role of: pulmonary hypertension, male gender, $\mathrm{VE} / \mathrm{VCO}_{2}$ slope and $\mathrm{VO}_{2}[\mathrm{~mL} /$ $/ \mathrm{kg} / \mathrm{min}$ ] (Table 6).

\section{Discussion}

A positive MTWA test was obtained in $17.3 \%$ of the analyzed patients, whereas an indeterminate result was obtained in $15.6 \%$ of them. Both of 
Table 3. Differences in demographic or clinical features between the subgroups defined by microvolt T-wave alternans (MTWA) result.

\begin{tabular}{|c|c|c|c|c|c|c|c|}
\hline & $\begin{array}{l}\text { Patients } \\
(n=179)\end{array}$ & $\begin{array}{c}\text { MTWA (+) } \\
(n=31)\end{array}$ & $\begin{array}{l}\text { MTWA (ind) } \\
\quad(n=28)\end{array}$ & $\begin{array}{l}\text { MTWA } \\
\text { (non-) } \\
(n=59)\end{array}$ & $\begin{array}{c}\text { MTWA } \\
(-) \\
(\mathrm{n}=120)\end{array}$ & $\begin{array}{c}\text { P: MTWA } \\
(+) \text { vs. } \\
\text { MTWA (-) }\end{array}$ & $\begin{array}{l}\text { P: MTWA } \\
\text { (non-) vs. } \\
\text { MTWA (-) }\end{array}$ \\
\hline Males & $\begin{array}{c}81 \\
(45.3 \%)\end{array}$ & $\begin{array}{c}24 \\
(77.4 \%)\end{array}$ & $\begin{array}{c}12 \\
(42.9 \%)\end{array}$ & $\begin{array}{c}36 \\
(61.0 \%)\end{array}$ & $\begin{array}{c}45 \\
(37.5 \%)\end{array}$ & 0.0002 & 0.005 \\
\hline $\begin{array}{l}\text { Age [years]; } \\
\text { median (min-max) }\end{array}$ & $\begin{array}{c}30 \\
(18-75)\end{array}$ & $\begin{array}{c}29 \\
(20-65)\end{array}$ & $\begin{array}{c}34 \\
(20-75)\end{array}$ & $\begin{array}{c}31 \\
(20-75)\end{array}$ & $\begin{array}{c}30 \\
(19-74)\end{array}$ & 0.982 & 0.325 \\
\hline $\begin{array}{l}\text { Body mass index } \\
{\left[\mathrm{kg} / \mathrm{m}^{2}\right] ; \text { median }} \\
(\mathrm{min}-\mathrm{max})\end{array}$ & $\begin{array}{c}21.8 \\
(15.6-38.0)\end{array}$ & $\begin{array}{c}22.3 \\
(15.8-33.8)\end{array}$ & $\begin{array}{c}21.2 \\
(15.6-38.1)\end{array}$ & $\begin{array}{c}21.8 \\
(15.6-38.1)\end{array}$ & $\begin{array}{c}21.8 \\
(15.7-37.2)\end{array}$ & 0.68 & 0.968 \\
\hline NYHA > I & $\begin{array}{c}56 \\
(31.3 \%)\end{array}$ & $\begin{array}{c}10 \\
(32.3 \%)\end{array}$ & $\begin{array}{c}16 \\
(57.1 \%)\end{array}$ & $\begin{array}{c}26 \\
(44.1 \%)\end{array}$ & $\begin{array}{c}30 \\
(25.0 \%)\end{array}$ & 0.339 & 0.007 \\
\hline $\begin{array}{l}\text { Blood oxygen } \\
\text { saturation [\%]; } \\
\text { median (min-max) }\end{array}$ & $\begin{array}{c}98 \\
(69-100)\end{array}$ & $\begin{array}{c}97 \\
(75-100)\end{array}$ & $\begin{array}{c}97.5 \\
(73-100)\end{array}$ & $\begin{array}{c}97 \\
(73-100)\end{array}$ & $\begin{array}{c}99 \\
(69-100)\end{array}$ & 0.009 & 0.0003 \\
\hline Operated & $\begin{array}{c}126 \\
(70.4 \%)\end{array}$ & $\begin{array}{c}23 \\
(74.2 \%)\end{array}$ & $\begin{array}{c}15 \\
(53.6 \%)\end{array}$ & $\begin{array}{c}38 \\
(64.4 \%)\end{array}$ & $\begin{array}{c}88 \\
(73.3 \%)\end{array}$ & 1.0 & 0.291 \\
\hline $\begin{array}{l}\text { Age at operation } \\
\text { [years]; median } \\
\text { (min-max) }\end{array}$ & $\begin{array}{c}5.0 \\
(0.5-21)\end{array}$ & $\begin{array}{c}4 \\
(0.5-17)\end{array}$ & $\begin{array}{c}7 \\
(0.5-21)\end{array}$ & $\begin{array}{c}3 \\
(0.5-21)\end{array}$ & $\begin{array}{c}3 \\
(0.5-16)\end{array}$ & 0.817 & 0.404 \\
\hline $\begin{array}{l}\text { Pulmonary } \\
\text { hypertension }\end{array}$ & $\begin{array}{c}11 \\
(6.1 \%)\end{array}$ & $\begin{array}{c}3 \\
(9.7 \%)\end{array}$ & $\begin{array}{c}7 \\
(25.0 \%)\end{array}$ & $\begin{array}{c}10 \\
(16.9 \%)\end{array}$ & $\begin{array}{c}1 \\
(0.8 \%)\end{array}$ & 0.03 & 0.00007 \\
\hline
\end{tabular}

Table 4. Differences in ventricular arrhythmia incidence between the subgroups defined by microvolt T-wave alternans (MTWA) result.

\begin{tabular}{|c|c|c|c|c|c|c|c|}
\hline & $\begin{array}{l}\text { Patients } \\
\text { ( } n=179)\end{array}$ & $\begin{array}{c}\text { MTWA } \\
(+) \\
(n=31)\end{array}$ & $\begin{array}{c}\text { MTWA } \\
\text { (ind) } \\
(n=28)\end{array}$ & $\begin{array}{l}\text { MTWA } \\
\text { (non-) } \\
(n=59)\end{array}$ & $\begin{array}{c}\text { MTWA } \\
(-) \\
\text { (n= 120) }\end{array}$ & $\begin{array}{c}\text { P: MTWA } \\
(+) \text { vs. } \\
\text { MTWA (-) }\end{array}$ & $\begin{array}{l}\text { P: MTWA } \\
\text { (non-) vs. } \\
\text { MTWA (-) }\end{array}$ \\
\hline VT & $12(6.7 \%)$ & $2(6.9 \%)$ & $7(25.0 \%)$ & $9(15.2 \%)$ & $3(2.5 \%)$ & 0.25 & 0.003 \\
\hline$n s V T / V P B>10 / h$ & $\begin{array}{c}40 \\
(22.3 \%)\end{array}$ & $\begin{array}{c}7 \\
(22.6 \%)\end{array}$ & $\begin{array}{c}13 \\
(68.4 \%)\end{array}$ & $\begin{array}{c}20 \\
(33.9 \%)\end{array}$ & $\begin{array}{c}20 \\
(16.7 \%)\end{array}$ & 0.439 & 0.02 \\
\hline VT/nsVT/VPB $>10 / \mathrm{h}$ & $\begin{array}{c}44 \\
(24.6 \%)\end{array}$ & $\begin{array}{c}8 \\
(25.8 \%)\end{array}$ & $\begin{array}{c}15 \\
(53.6 \%)\end{array}$ & $\begin{array}{c}23 \\
(39.0 \%)\end{array}$ & $\begin{array}{c}21 \\
(17.5 \%)\end{array}$ & 0.312 & 0.003 \\
\hline
\end{tabular}

MTWA (+) - positive result; MTWA (ind) - indeterminate result; MTWA $(-)$ - negative result; MTWA (non-) - non-negative result; VPB $\geq 10 / h$ - excessive ventricular ectopy; nsVT — non-sustained ventricular tachycardia; VT — sustained ventricular tachycardia

these values are significantly higher than among healthy subjects with one positive test $(2.3 \%$ of the group) and none indeterminate MTWA result [22]. The incidence of MTWA (+) observed by us was comparable to the values obtained in our previous study regarding adults with congenital defect characterized by the right ventricle pathology or univentricular heart, where the incidence of positive, as well as indeterminate MTWA accounted for $17.6 \%$ each. As far as pediatric CHD population is concerned, Alexander et al. [17] described this phenomenon in $14 \%$ of patients, whereas Cheung et al. [18] described it in $14.2 \%$ of children after tetralogy of Fallot repair.
There is, however, a great difference between the results obtained by our team and the trials conducted on patients with advanced ischemic/ /nonischemic heart failure, in which the incidence of MTWA (+) reached 37-52\% [13-16]. Although not all researchers confirmed the prognostic value of the MTWA phenomenon, the analysis of numerous studies demonstrated its significant diagnostic role excluding the probability of SCD [10-12, 15]. On the other hand, positive and indeterminate MTWA results were proved to have similar prognostic value, therefore being qualified together as non-negative (abnormal) [10, 13-16]. The abnormal MTWA was seen in $32.9 \%$ of our patients, which 
Table 5. Differences in the chosen cardiopulmonary parameters and serum B-type natriuretic peptide level between the subgroups defined by microvolt T-wave alternans (MTWA) result.

\begin{tabular}{|c|c|c|c|c|c|c|c|}
\hline & $\begin{array}{l}\text { Patients } \\
(n=174)\end{array}$ & $\begin{array}{c}\text { MTWA (+) } \\
(n=30)\end{array}$ & $\begin{array}{l}\text { MTWA (ind) } \\
(n=27)\end{array}$ & $\begin{array}{c}\text { MTWA } \\
(\text { non-) } \\
(n=57)\end{array}$ & $\begin{array}{c}\text { MTWA } \\
(-) \\
(n=117)\end{array}$ & $\begin{array}{c}\text { P: MTWA } \\
(+) \text { vs. } \\
\text { MTWA (-) }\end{array}$ & $\begin{array}{l}\text { P: MTWA } \\
\text { (non-) vs. } \\
\text { MTWA (-) }\end{array}$ \\
\hline $\begin{array}{l}\text { BNP }[\mathrm{pg} / \mathrm{mL}] ; \\
\text { median (min-max) }\end{array}$ & $\begin{array}{c}39.9 \\
(6.2-598.4)\end{array}$ & $\begin{array}{c}35.8 \\
(9.2-195.4)\end{array}$ & $\begin{array}{c}60.4 \\
(25.1-343.5)\end{array}$ & $\begin{array}{c}50.8 \\
(9.2-343.5)\end{array}$ & $\begin{array}{c}35.5 \\
(6.2-5984)\end{array}$ & 0.398 & 0.03 \\
\hline $\mathrm{VO}_{2}[\mathrm{~mL} / \mathrm{kg} / \mathrm{min}]$ & $25.2 \pm 6.6$ & $23.8 \pm 5.1$ & $22.2 \pm 6.6$ & $23.1 \pm 5.9$ & $26.3 \pm 6.7$ & 0.03 & 0.002 \\
\hline $\begin{array}{l}\% \mathrm{VO}_{2}[\mathrm{~mL} / \mathrm{kg} / \mathrm{min}] \\
\text { median }(\mathrm{min}-\max )\end{array}$ & $\begin{array}{c}64.0 \\
(28.0-118.0)\end{array}$ & $\begin{array}{c}60.5 \\
(36.0-78.0)\end{array}$ & $\begin{array}{c}63.0 \\
(28.0-86.0)\end{array}$ & $\begin{array}{c}61.0 \\
(28.0-86.0)\end{array}$ & $\begin{array}{c}64.5 \\
(30.0-118.0)\end{array}$ & 0.01 & 0.01 \\
\hline $\begin{array}{l}\mathrm{VO}_{2}[\mathrm{~L} / \mathrm{min}] \\
\text { median }(\min -\max )\end{array}$ & $\begin{array}{c}1.5 \\
(0.5-2.8)\end{array}$ & $\begin{array}{c}1.6 \\
(0.5-2.6)\end{array}$ & $\begin{array}{c}1.2 \\
(0.5-2.9)\end{array}$ & $\begin{array}{c}1.5 \\
(0.5-2.9)\end{array}$ & $\begin{array}{c}1.6 \\
(0.7-2.8)\end{array}$ & 0.883 & 0.088 \\
\hline $\begin{array}{l}\% \mathrm{VO}_{2}[\mathrm{~L} / \min ] ; \\
\text { median (min-max) }\end{array}$ & $\begin{array}{c}64.0 \\
(26.0-108.0)\end{array}$ & $\begin{array}{c}57.5 \\
(33.0-78.0)\end{array}$ & $\begin{array}{c}59.0 \\
(31.0-106.0)\end{array}$ & $\begin{array}{c}59.0 \\
(31.0-106.0)\end{array}$ & $\begin{array}{c}66.0 \\
(26.0-108.0)\end{array}$ & 0.003 & 0.002 \\
\hline $\begin{array}{l}\mathrm{VE} / \mathrm{VCO}_{2} \text { slope; } \\
\text { median (min-max) }\end{array}$ & $\begin{array}{c}32.0 \\
(21-74)\end{array}$ & $\begin{array}{c}33.5 \\
(25-74)\end{array}$ & $\begin{array}{c}39.0 \\
(26-66)\end{array}$ & $\begin{array}{c}36.0 \\
(25-74)\end{array}$ & $\begin{array}{c}31.0 \\
(21-58)\end{array}$ & 0.247 & 0.01 \\
\hline $\begin{array}{l}\text { RER; } \\
\text { median (min-max) }\end{array}$ & $\begin{array}{c}1.02 \\
(0.95-1.19)\end{array}$ & $\begin{array}{c}1.01 \\
(1.0-1.09)\end{array}$ & $\begin{array}{c}1.01 \\
(1.0-1.13)\end{array}$ & $\begin{array}{c}1.01 \\
(1.0-1.13)\end{array}$ & $\begin{array}{c}1.02 \\
(0.95-1.19)\end{array}$ & 0.167 & 0.141 \\
\hline
\end{tabular}

MTWA (+) - positive result; MTWA (ind) — result indeterminate due to patient factors; MTWA (-) — negative result; MTWA (non-) - non-negative result; BNP - B-type natriuretic peptide; $\mathrm{VO}_{2}$ - peak oxygen uptake on exercise; $\mathrm{VE} / \mathrm{VCO}_{2}$ slope - ventilation/carbon dioxide slope, RER - respiratory exchange ratio

Table 6. Factors increasing probability of abnormal microvolt T-wave alternans (MTWA) in uni- and multivariate logistic regression analysis.

\begin{tabular}{|c|c|c|c|c|}
\hline & \multicolumn{2}{|c|}{ Univariate analysis } & \multicolumn{2}{|c|}{ Multivariate analysis } \\
\hline & OR $(95 \% \mathrm{CI})$ & $\mathbf{P}$ & OR $(95 \% \mathrm{Cl})$ & $\mathbf{P}$ \\
\hline Pulmonary hypertension [\%] & $24.29(2.97-198.85)$ & 0.003 & $13.62(1.24-149.5)$ & 0.03 \\
\hline VT [\%] & 7.31 (1.88-28.49) & 0.004 & $8.4(0.69-102.04)$ & 0.095 \\
\hline VT/nsVT/VPB $\geq 10 / \mathrm{h}[\%]$ & $3.01(1.48-6.12)$ & 0.002 & $1.54(0.04-55.1)$ & 0.814 \\
\hline Males [\%] & $2.61(1.37-4.97)$ & 0.003 & $10.39(3.55-30.42)$ & 0.00002 \\
\hline NYHA > I [\%] & $2.06(1.21-3.49)$ & 0.007 & $1.27(0.47-3.35)$ & 0.636 \\
\hline VE/ $\mathrm{VCO}_{2}$ slope & $1.07(1.02-1.11)$ & 0.002 & $1.07(1.00-1.14)$ & 0.045 \\
\hline$\% \mathrm{VO}_{2}[\mathrm{~mL} / \mathrm{kg} / \mathrm{min}]$ & $0.97(0.94-0.99)$ & 0.007 & $1.01(0.95-1.08)$ & 0.711 \\
\hline$\% \mathrm{VO}_{2}[\mathrm{~L} / \mathrm{min}]$ & $0.97(0.94-0.99)$ & 0.005 & $1.00(0.95-1.06)$ & 0.929 \\
\hline $\mathrm{SO}_{2}[\%]$ & $0.93(0.88-0.98)$ & 0.006 & $1.04(0.94-1.15)$ & 0.379 \\
\hline $\mathrm{VO}_{2}[\mathrm{~mL} / \mathrm{kg} / \mathrm{min}]$ & $0.92(0.87-0.97)$ & 0.003 & $0.89(0.80-0.99)$ & 0.04 \\
\hline
\end{tabular}

OR — odds ratio; $\mathrm{Cl}$ — confidential interval; VT — sustained ventricular tachycardia; VPB $\geq 10 / \mathrm{h}$ - excessive ventricular ectopy; nsVT — non-sustained ventricular tachycardia; NYHA — New York Heart Association; VE $/ \mathrm{VCO}_{2}$ slope — ventilation/carbon dioxide slope; VO ${ }_{2}$ - peak oxygen uptake on exercise, $\mathrm{SO}_{2}-$ oxygen blood saturation

was significantly less than the values presented in studies quoted above regarding ischemic and nonischemic heart failure (67-73\% of patients). It is presumably the younger age of our patients, who were mostly in their forties, whereas subjects enrolled in the mentioned trials reached their $6^{\text {th }}$ or $7^{\text {th }}$ decade of life, that contributed to such a difference in MTWA incidence. Moreover, the functional status of our congenital heart diseases patients was also much better, with $85 \%$ being in NYHA class I.
Our study showed that a previous history of malignant and potentially malignant arrhythmia was significantly more frequent in patients with abnormal MTWA compared with the MTWA (-) group. However, the we did not confirm the relationship of that arrhythmia reported in the past with abnormal MTWA, which is congruent with the outcome of the analysis conducted in pediatric CHD population [17].

Although the ESC and AHA experts [6, 9] recommend ambulatory ECG monitoring as 
a method of SCD risk stratification in patients with structurally diseased hearts (which undeniably concerns our study group), the prognostic role of malignant arrhythmia episodes observed during this test has never been confirmed in the general population. It is known, however, that arrhythmia increases mortality among subjects with advanced heart failure simultaneously taking part in heart failure pathogenesis. On malignant arrhythmia risk stratification algorithm the analysis of ambulatory ECG monitoring is justifiable when combined with other clinical findings increasing this risk. A comprehensive analysis by Cutler and Rosenbaum [10], regarding the papers published in the last decade, undeniably demonstrated an algorithm considering a few carefully combined ventricular arrhythmia risk factors in patients with ischemic or nonischemic cardiomyopathy to enable more accurate qualification for ICD in primary prevention of SCD, exceeding even outcomes of implantations made in secondary prophylaxis. It seems reasonable to apply these rules also in CHD population.

Therefore, we expanded the analysis to determine which demographical and clinical findings of possible prognostic value for SCD are observed in subjects with abnormal MTWA. The association between a patient's age and MTWA result was not confirmed, although in the general population the risk of SCD increases significantly after the age of 35 [6]. In the analyzed subjects who have been affected with the disease since birth, it is rather the severity of a given defect that worsens a patient's prognosis. Also the fact of undergoing potentially beneficial cardio-surgery turned out to have no prognostic value, as repairs are usually performed in advanced cardiac anomalies and remaining scars may be the origin of arrhythmia [1-5]. Our study showed in turn the predominance of males among MTWA (non-) patients with male gender being also a factor that increases the probability of this phenomenon. This confirms the observation that men die more often due to SCD than women [6]. In the CHD population male gender was proved to be a risk factor of life-threatening arrhythmia [23, 24], as well as of total mortality [2].

Our study demonstrated worse exercise tolerance in patients with abnormal MTWA when compared to the MTWA (-) group and their functional status was more often impaired (NYHA $>$ I). It is proved that deterioration in NYHA classification has an adverse prognostic value for SCD in patients with congenital heart anomaly [7, 25, 26]. This association might be due to the heart failure-related hypercatecholaminemia that increases the risk of ventricular arrhythmia, simultaneously confirming the rationale for including MTWA into SCD risk stratification in the analyzed population [27]. However, our study neither proved higher NYHA class nor higher serum BNP concentration to increase the probability of abnormal MTWA. We confirmed the prognostic value of cardiopulmonary parameters: ventilation-carbon dioxide production relationship (VE/ $/ \mathrm{VCO}_{2}$ slope) and peak oxygen consumption $\left(\mathrm{VO}_{2}[\mathrm{~mL} / \mathrm{kg} / \mathrm{min}]\right)$. A predictive role of the latter parameter for ventricular arrhythmia in CHD population was also proved by other authors [23].

Other factors contributing to heart failure pathogenesis in patients with that heart anomaly are elevated pulmonary and decreased blood saturation, often coexisting, hence difficult to be analyzed separately [26, 27]. We observed these 2 clinical features more often in patients with abnormal MTWA, though only pulmonary hypertension was confirmed to increase the probability of this phenomenon. Sub-pulmonary ventricle pressure overload along with cyanosis-related tissue hypoxia, as well as segmental dilation and tortuosity of the coronary arteries typical for increased blood viscosity lead to the myocardial fibrosis creating a substrate for ventricular arrhythmia [28, 29].

Similarly to the general cardiologic population [13-16], among patients with CHD the abnormal MTWA was observed more frequent in those with advanced heart failure, already proved to be linked to ventricular arrhythmia. The parameters determining the cardiac impairment in this anatomically diversified population turned out to be the factors increasing probability of non-negative MTWA. Although the unquestionable relationship between this phenomenon and malignant or potentially malignant arrhythmia was not stated, the obtained results give the rationale for applying MTWA test in SCD risk stratification algorithm for CHD patients. Further studies are required to confirm the value of this diagnostic tool.

\section{Limitations of the study}

The main methodological limitations of our study are the lack of prospective analysis and the limited number of patients. A smaller group size than in ischemic and nonischemic heart failure trials is characteristic for this unique population and is seen in the majority of CHD studies. The number of patients, as well as the incidence of potential end points determine the follow-up period, which, according to the statistical rules, should last more than 10 years and has been already started by our team $[8,30]$. 


\section{Conclusions}

The incidence of abnormal MTWA among patients with congenital heart defects is higher than in healthy subjects. The probability of this phenomenon increases in patients with clinical findings possibly conducive to lethal arrhythmia including heart failure and pulmonary hypertension, as well as in males. The confirmation of MTWA potential role in SCD risk stratification in this population requires further investigation.

\section{Conflict of interest: none declared}

\section{References}

1. Koyak Z, Harris L, De Groot JR et al. Sudden cardiac death in adult ongenital heart disease. Circulation, 2012; 126: 1944-1954 .

2. Verheugt CL, Uiterwaal CS, van der Velde ET et al. Mortality in adult congenital heart disease. Eur Heart J, 2010; 31: 1220-1229.

3. Oechslin EN, Harrison DA, Connelly MS, Webb GD, Siu SC. Mode of death in adults with congenital heart disease. Am J Cardiol, 2000; 86: 1111-1116.

4. Trojnarska O, Grajek S, Katarzyński S, Kramer L. Predictors of mortality in adult patients with congenital heart disease. Cardiol J, 2009; 16: 341-347.

5. Triedman JK. Should patients with congenital heart disease and a systemic ventricular ejection fraction less than $30 \%$ undergo prophylactic implantation of an ICD? Implantable cardioverter defibrillator implantation guidelines based solely on left ventricular ejection fraction do not apply to adults with congenital heart disease. Circ Arrhythm Electrophysiol, 2008; 1: 307-316.

6. Zipes DP, Camm AJ, Borggrefe M et al. ACC/AHA/ESC 2006 guidelines for management of patients with ventricular arrhythmias and the prevention of sudden cardiac death: A report of the ACC/AHA Task Force and the ESC Committee for Practice Guidelines developed in collaboration with the EHRA and HRS. Circulation, 2006; 114: e385-e484.

7. Baumgartner H, Bonhoeffer P, De Groot NM et al. ESC guidelines for the management of grown-up congenital heart disease (new version 2010). The task force on the management of grownup congenital heart disease of the European Society of Cardiology (ESC). Eur Heart J, 2010; 31: 2915-2957.

8. Walsh EP, Cecchin F. Arrhythmias in adult patients with congenital heart disease. Circulation, 2007; 115: 534-545.

9. Goldberger JJ, Cain ME, Hohnloser SH et al. AHA/ACC/HRS scientific statement on noninvasive risk stratification for identifying patients at risk for sudden cardiac death. Circulation, 2008; 118: 1497-1518.

10. Cutler MJ, Rosenbaum DS. Risk stratification for sudden cardiac death: is there a clinical role for T wave altenans? Heart Rhythm, 2009; 6: S56-S61.

11. Bloomfield DM, Hohnloser SH, Cohen RJ. Interpretation and classification of microvolt T-wave alternans tests. J Cardiovasc Electrophysiol, 2002; 13: 502-512.

12. Kaufman ES, Bloomfield DM, Steinman RC et al. "Indeterminate" microvolt $\mathrm{T}$ wave alternans tests predict high risk of death or sustained ventricular arrhythmias in patients with left ventricular dysfunction. J Am Coll Cardiol, 2006; 48: 1399-1404.

13. Costantini O, Hohnnloser SH, Kirk MM et al. The ABCD (Alternans Before Cardioverter Defibrillator) Trial: Strategies using
T-wave alternans to improve efficiency of sudden cardiac death prevention. J Am Coll Cardiol, 2009; 53: 471-479.

14. Van der Avoort CJ, Filion KB, Dendukuri N, Brophy JM. Microvolt T-wave alternans as a predictor of mortality and severe arrhythmias in patients with left-ventricular dysfunction: A systemic review and meta-analysis. BMC Cardiovascular Disord, 2009; 9: 5.

15. Verrier RL, Klingenheben T, Malik M et al. Microvolt T-Wave Alternans Physiological Basis, Methods of Measurement,and Clinical Utility - Consensus Guideline by International Society for Holter and Noninvasive Electrocardiology. J Am Coll Cardiol, 2011; 58: 1309-1324.

16. Gehi AK, Stein RH, Metz LD, Gomez A. Microvolt T-wave alternans for the risk stratification of ventricular tachyarrhythmic events. A meta-analysis. J Am Coll Cardiol, 2005; 46: 75-82.

17. Alexander ME, Cecchin F, Huang KP, Berul CI. Microvolt T-wave alternans with exercise in pediatrics and congenital heart disease: Limitations and predictive value. Pacing Clin Electrophysiol, 2006; 29: 733-741.

18. Cheung MM, Weintraub RG, Cohen RJ, Karl TR, Wilkinson JL, Davis AM. T wave alternans threshold late after tetralogy of Fallot. J Cardiovasc Electrophysiol, 2002; 13: 657-661.

19. Cieplucha A, Trojnarska O, Bartczak A, Kramer L, Grajek S. Microvolt $\mathrm{T}$ wave alternans in adults with congenital heart diseases characterized by right ventricle pathology or single ventricle physiology: A case control study. BMC Cardiovasc Disord, 2013; 13: 26.

20. Galie N, Hoeper MM, Humbert M et al. Guidelines for the diagnosis and treatment of pulmonary hypertension. The Task Force for the diagnosis and treatment of pulmonary hypertension of the ESC and ERS, endorsed by the ISHLT. Eur Heart J, 2009; 30: 2493-2537.

21. Wasserman K, Hansen JE, Sue DY. Principles of exercise testing and interpretation. Lea and Febiger, Philadelphia, PA 1986.

22. Weber S, Thillmanns H, Waldecker B. Prevalence of TWA in healthy subjects. Pacing Clin Electrophysiol, 2003; 26: 49-52.

23. Tsai SF, Chan DP, Ro PS, Boettner B, Daniels CJ. Rate of inducible ventricular arrhythmia in adults with congenital heart disease. Am J Cardiol, 2010; 106: 730-736.

24. Khairy P, Aboulhosn J, Gurvitz MZ et al. Arrhythmia burden in adults with surgically repaired tetralogy of Fallot. A multiinstitutional study. Circulation, 2010; 122: 868-875.

25. Schwerzmann M, Salehian O, Harris L et al. Ventricular arrhythmias and sudden death in adults after a Mustard operation for transposition of the great arteries. Eur Heart J, 2009; 30: 1873-1879.

26. Diller GP, Dimopoulos K, Broberg CS et al. Presentation, survival prospects, and predictors of death in Eisenmenger syndrome: a combined retrospective and case-control study. Eur Heart J, 2006; 27: 1737-1742.

27. Trojnarska O, Gwizdała A, Katarzyński S et al. The BNP concentration and exercise capacity assessment with cardiopulmonary stress exercise test in cyanotic patients with congenital heart diseases. Int J Cardiol, 2010; 139: 241-247.

28. Diller GP, Giardini A, Dimopoulos K et al. Predictors of morbidity and mortality in contemporary Fontan patients: Results from a multicenter study including cardiopulmonary exercise testing in 321 pts. Eur Heart J, 2010; 31: 3073-3083.

29. Khairy P, Harris L, Landzberg MJ et al. Sudden death and defibrillators in transposition of the great arteries with intra-atrial baffles. A multicenter study. Circ Arrhythmia Electrophysiol, 2008; 1: 250-257.

30. Silka MJ, Bar-Cohen Y. Should patients with congenital heart disease and a systemic ventricular ejection fraction less than $30 \%$ undergo prophylactic implantation of an ICD? Patients with congenital heart disease and a systemic ventricular ejection fraction less than $30 \%$ should undergo prophylactic implantation of an implantable cardioverter defibrillator. Circ Arrhythm Electrophysiol, 2008; 1: 298-306. 